WHOI -81-22

BAROTROPIC RESPONSE TO COOLING

by

Henry Stomme 1

and

George Veronis

WOODS HOLE OCEANOGRAPHIC INSTITUTION

Woods Hole, Massachusetts 02543

April 1981

TECHNICAL REPORT

Prepared for the Office of Naval Research under Contract N00014-79-C-0671 and for the National Science Foundation under Grants OCE 77-19451 and OCE 78-18460.

Reproduction in whole or in part is permitted for any purpose of the United States Govermment. In citing this report in a bibliogrophy, the reference given should be to: Joumal of Geophysical Research 85(C11): -6661-6666 (November 20, 1980).

Approved for public release; distribution unlimited.

Approved for Distribution: Valentine Worthington, Chairman Department of Physical Oceanography 


\title{
Barotropic Response to Cooling
}

\author{
HENRY STOMMEL
}

Woods Hole Oceanographic Institution, Woods Hole, Massachusetts 02543

\author{
GEORGE VERONIS
}

Department of Geology and Geophysics, Yale University, New Haven, Connecticut 06520

\begin{abstract}
Imposed horizontal density differences in a nonrotating fluid generate vertical circulation which has vanishing vertically integrated transports. When the system is rotating, geostrophic velocities can balance the density differences and the vertically integrated transports need not vanish locally. In a two-layer fluid, finite amplitude disturbances lead to barotropic flows that have the same direction as the velocity in the layer that thickens as a result of the disturbance. Specific calculations are carried out for the geostrophic adjustment model in situations that approximate those in which $18^{\circ}$ water is formed south of the Gulf Stream. The upper layer transport that results from sudden cooling (as simulated by density differences that are initially unbalanced geostrophically) is in the same direction as the Gulf Stream transport and comparable to it in magnitude. A lower level transport of the same magnitude flows in the opposite direction with a maximum value about an internal radius of deformation to the right of that of the upper layer. The barotropic transport is about $1 / 5$ as large and flows downstream in the Gulf Stream and upstream to the right of the Gulf Stream.
\end{abstract}

\section{INTRODUCTION}

It is often assumed that the response of a stratified ocean to sudden cooling - as in the northwestern Mediterranean, the Labrador Sea and $18^{\circ}$ water south of the Gulf Stream-is baroclinic, i.e., the vertically integrated flow vanishes. There is ample evidence to support such an assumption in certain simplified systems. For example, suppose that a vertical barrier separating a layer of dense water on the left from less dense water on the right is suddenly removed. Light water will flow to the left near the surface, dense water will flow to the right near the bottom and eventually a stably stratified, two-layer system will be established. During the adjustment the vertically integrated flow at any position will vanish.

If, however, the entire system is rotating, only part of the initial potential energy is released by penetration to the right and left. The remainder will support a geostrophic flow parallel to the plane of the barrier. We show in the next section that this induced horizontal flow generally has a nonvanishing vertical average at each point.

As a second example, consider the flow in a stably stratified, two-layer, rotating fluid which is perturbed infinitesimally. With subscripts 1 and 2 referring to upper and lower layers, respectively, the conservation of potential vorticity of the final equilibrium state is expressed as

$$
\begin{aligned}
& \frac{\zeta_{1}+f}{h_{1}}=\frac{f}{H_{1}} \\
& \frac{\zeta_{2}+f}{h_{2}}=\frac{f}{H_{2}}
\end{aligned}
$$

where $f$ is the Coriolis parameter, $\zeta_{i}$ are the vertical velocities, and $h_{i}$ and $H_{i}$ are the perturbed and unperturbed depths of the two layers. These equations yield

$$
\begin{aligned}
& H_{1} \zeta_{i}+f H_{1}=f h_{1} \\
& H_{2} \zeta_{2}+f H_{2}=f h_{2}
\end{aligned}
$$

so

Copyright (c) 1980 by the American Geophysical Union.

$$
H_{1} \zeta_{1}+H_{2} \zeta_{2}+\left(H_{1}+H_{2}\right) f=\left(h_{1}+h_{2}\right) f
$$

If the fluid is confined between rigid level boundaries at top and bottom, the total depths $H_{1}+H_{2}$ and $h_{1}+h_{2}$ are equal and the result is

$$
H_{1} \zeta_{1}+H_{2} \zeta_{2}=0
$$

Therefore the vertically integrated vorticities vanish for linearized flows. If the velocities vanish at some point (say, at infinity) the last equation can be integrated horizontally from that point, and we conclude that the vertically integrated transports vanish everywhere for linearized flows.

For flows of finite amplitude the vertically integrated vorticities become

$$
\begin{aligned}
& h_{1} \zeta_{1}+h_{1} f=f \frac{h_{1}{ }^{2}}{H_{1}} \\
& h_{2} \zeta_{2}+h_{2} f=f \frac{h_{2}{ }^{2}}{H_{2}}
\end{aligned}
$$

and the total integrated vorticity is

$$
h_{1} \zeta_{1}+h_{2} \zeta_{2}=f\left(\frac{1}{H_{1}}+\frac{1}{H_{2}}\right)\left(\Delta h_{1}\right)^{2}
$$

where $\Delta h_{1}=h_{1}-H_{1}=-h_{2}+H_{2}$ measures the distortion of the interface from its equilibrium level. Hence the vertically integrated vorticity does not vanish.

If we consider the simple case where the flow is independent of $y$, we have $\zeta_{1}=\partial v_{1} / \partial x$ and $\zeta_{2}=\partial v_{2} / \partial x$. Furthermore, if the flow vanishes at $x=-\infty$, we can integrate $(1 a)$ and $(1 b)$ from $-\infty$ to $x$ to obtain

$$
\begin{aligned}
& v_{1}=\frac{f}{H_{1}} \int_{-\infty}^{x} \Delta h_{1} d f \\
& v_{2}=\frac{-f}{H_{2}} \int_{-\infty}^{x} \Delta h_{1} d f
\end{aligned}
$$

Then the vertical integrals of $v$ over each layer added together yield the total barotropic transport

$$
T_{B} \equiv h_{1} v_{1}+h_{2} v_{2}=f\left(\frac{1}{H_{1}}+\frac{1}{H_{2}}\right) \Delta h_{1} \int_{-\infty}^{x} \Delta h_{1} d x
$$




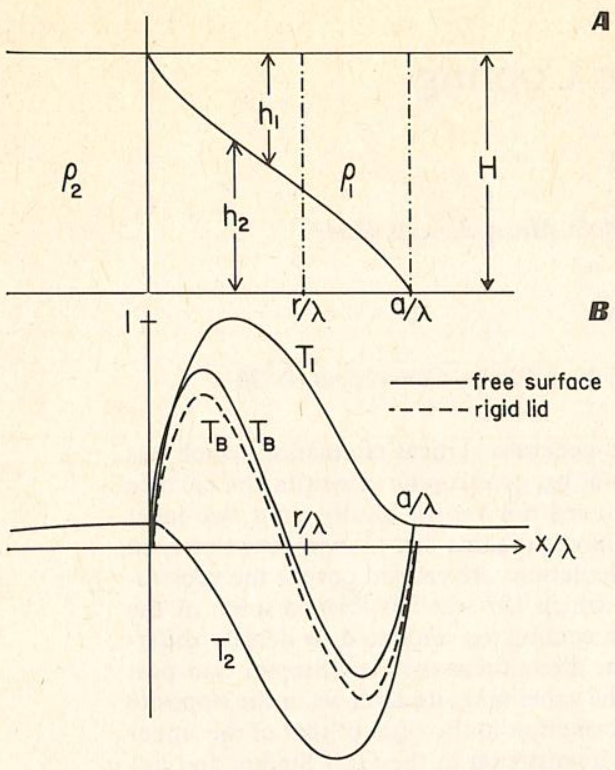

Fig. 1. (a) The shape of the interface for fluids of densities $\rho_{1}$ and $\rho_{2}\left(>\rho_{1}\right)$ initially separated by a vertical barrier. The upper layer penetrates to the left to the point where its thickness, $h_{1}$, vanishes. With that point as the origin the lower layer penetrates to the point $x=a$, where $a=0.107 \lambda_{2}$. Initially the layers of fluid were separated by a vertical barrier at $x=r$ where $r=0.0642 \lambda_{2}$. The abscissa is in units of $x / \lambda$. (b) Upper layer, lower layer, and total (barotropic) transports marked by $T_{1}, T_{2}$, and $T_{B}$, respectively, for the free surface case of Figure $1 a$. The dashed line shows $T_{B}$ for the case with a rigid lid. Transports are given relative to maximum value of $T_{B}$.

For $\Delta h_{1}>0\left(h_{1}>H_{1}\right)$ we obtain $v_{1}>0$ and $v_{2}<0$ so $T_{B}$ has the same sign as $v_{1}$. For $\Delta h_{1}<0\left(h_{2}>H_{2}\right)$ we have $v_{1}<0$ and $v_{2}>0$ so $T_{B}$ and $v_{2}$ have the same sign. Thus the barotropic transport will have the same sign as the velocity in the layer that has become thicker.

The foregoing suggests that finite amplitude motions generated by initially unbalanced, horizontal density difference in a rotating fluid lead to nonvanishing barotropic transports. We shall explore this question further in the following sections and obtain quantitative estimates for specific flows. The physical situation is the adjustment problem, though we have in mind those regions of sudden cooling of surface waters mentioned at the outset. Thus we assume that the cooling takes place over a time scale short compared to the time required to set up the adjusted flows. Our conclusions would have to be modified if significant adjustment were to take place during the cooling phase.

The adjustment problem has been studied by many people since Rossby [1938] suggested the process as an important one. The analyses presented below contain no new analytical results. Our aim is simply to investigate the degree to which the process leads to barotropic flows and to obtain quantitative estimates of the barotropic transports.

In section 2 the adjustment of two masses of fluid initially separated by a vertical barrier is analyzed for the cases where the top surface is free and rigid. We show that the free surface problem is essentially the rigid lid case with a superposed barotropic mode due to the distortion of the top surface. This result is used to justify the assumption of a rigid lid in the more complicated cases of sections 3 and 4 . Quantitative results with oceanic values for the parameters are given in the latter two problems.

\section{VERTICAL BARRIER}

We explore the effect of a free surface by considering two homogeneous layers of water initially separated by a vertical barrier with water of density $\rho_{1}$ and depth $H_{1}$ to the right and water that has been cooled to density $\rho_{2}=\rho_{1}+\Delta \rho$ and with depth $H_{2}$ to the left. Initially, the mass on each side is the same so $H_{1} \rho_{1}=H_{2} \rho_{2}$. Conditions are assumed uniform parallel to the barrier $\left(\partial_{y} \equiv 0\right)$.

After adjustment there will be light water over dense water as shown in Figure $1 a$. We place the origin of the $x$ axis at the point where the depth $h_{1}$ of the upper layer vanishes after ad-justment. In this coordinate system the location $x=r$ of the original barrier and the point $x=a$ at which the lower layer depth $h_{2}$ vanishes must be determined.

The adjusted, geostrophic state will consist of three regions. Dense fluid occupies the entire depth to the left of $x=0$ so the pertinent equations are $(1 b)$ with $\zeta_{2}=\partial v_{2} / \partial x$ and geostrophic balance given by

$$
f v_{2}=g \frac{\partial h_{2}}{\partial x}
$$

The solution for $x \leq 0$ is

$$
\begin{gathered}
h_{2}=H_{2}\left[1+A_{L} e^{x / \lambda_{2}}\right] \\
v_{2}=f \lambda_{2} A_{L} e^{x / \lambda_{2}}
\end{gathered}
$$

where $\lambda_{2}{ }^{2}=g H_{2} / f^{2} . A_{L}$ is to be determined.

Light fluid occupies the entire depth, $h_{1}$, to the right of $x=$ $a$ and the pertinent equations are ( $1 a)$ with $\zeta_{1}=\partial v_{1} / \partial x$ and geostrophic balance given by

$$
f v_{1}=g \partial h_{1} / \partial x
$$

The solution for $x \geq a$ is

$$
\begin{gathered}
h_{1}=H_{1}\left(1+A_{R} e^{-x / \lambda_{1}}\right) \\
v_{1}=-f \lambda_{1} A_{R} e^{-x / \lambda_{1}}
\end{gathered}
$$

where $\lambda_{1}^{2}=g H_{1} / f^{2} . A_{R}$ is to be determined.

Between $x=0$ and $x=a$ both fluids are present and the geostrophic equations become

$$
\begin{gathered}
f v_{1}=g \frac{\partial}{\partial x}\left(h_{1}+h_{2}\right) \\
f v_{2}=g \frac{\partial}{\partial x}\left(\frac{\rho_{1}}{\rho_{2}} h_{1}+h_{2}\right)
\end{gathered}
$$

These equations can be combined with $(1 a),(1 b)$ and $\zeta_{1}=$ $\partial v_{1} / \partial x, \zeta_{2}=\partial v_{2} / \partial x$, to yield the following fourth-order equation in $h_{1}$

$$
\lambda_{i}{ }^{2} \lambda_{2}{ }^{2} h_{1 x x x x}-\left(\lambda_{2}{ }^{2}+\lambda_{1}{ }^{2}\right) h_{1 x x}+h_{1}=H_{1}
$$

where $\lambda_{i}^{2}=\left(\Delta \rho / \rho_{2}\right)\left(g H_{1} / f^{2}\right)$.

The solution for $0 \leq x \leq a$ is

$$
\begin{aligned}
& h_{1}=H_{1}\left[1+A_{1} \sinh X_{1}+A_{2} \cosh X_{1}\right. \\
& \left.+A_{3} \sinh X_{2}+A_{4} \cosh X_{2}\right] \\
& h_{2}=H_{2}\left\{1+\left(1-\epsilon-\epsilon D_{1}^{2}\right)\left[A_{1} \sinh X_{1}+A_{2} \cosh X_{1}\right]\right. \\
& \left.+\left(1-\epsilon-\epsilon D_{2}^{2}\right)\left[A_{3} \sinh X_{2}+A_{4} \cosh X_{2}\right]\right\} \\
& v_{1}=f \lambda_{2}\left\{\left(2-\epsilon-\epsilon D_{1}^{2}\right) D_{1}\left[A_{1} \cosh X_{1}+A_{2} \sinh X_{1}\right]\right.
\end{aligned}
$$




$$
\begin{aligned}
&+\left.\left(2-\epsilon-\epsilon D_{2}{ }^{2}\right) D_{2}\left[A_{3} \cosh X_{2}+A_{4} \sinh X_{2}\right]\right\} \\
& v_{2}=f \lambda_{2}\left\{\left(2-2 \epsilon-\epsilon D_{1}{ }^{2}\right) D_{1}\left[A_{1} \cosh X_{1}+A_{2} \sinh X_{1}\right]\right. \\
&\left.+\left(2-2 \epsilon-\epsilon D_{2}{ }^{2}\right) D_{2}\left[A_{3} \cosh X_{2}+A_{4} \sinh X_{2}\right]\right\}
\end{aligned}
$$

where $\epsilon=\Delta \rho / \rho_{2}, X_{1}=D_{1} x / \lambda_{2}, X_{2}=D_{2} x / \lambda_{2}$ and the quantities $D_{i}$ are the functions of $\epsilon$ given by

$$
D_{i}=\left\{\frac{2-\epsilon \pm\left[(2-\epsilon)^{2}-4 \epsilon(1-\epsilon)\right]^{1 / 2}}{2 \epsilon}\right\}^{1 / 2}
$$

The values $A_{1}, A_{2}, A_{3}, A_{4}$ are to be determined.

To obtain the six values of the $A$ 's and the value for $a$, we use the following conditions:

1. $h_{1}=0$ at $x=0$ in (9)

2. $h_{2}=0$ at $x=a$ in (10)

3. $h_{2}$ from (3) equals $h_{2}$ from (10) at $x=0$

4. $h_{1}$ from (6) equals $h_{1}$ from (9) at $x=a$

5. $v_{2}$ from (4) equals $v_{2}$ from (12) at $x=0$

6. $v_{1}$ from (6) equals $v_{1}$ from (11) at $x=a$

7. the volumes of the two fluids must be conserved; hence

$$
\int_{-\infty}^{r} H_{2} d x=\int_{-\infty}^{a} h_{2} d x
$$

and

$$
\int_{r}^{\infty} H_{1} d x=\int_{0}^{\infty} h_{1} d x
$$

The value of $r$ can be eliminated from these two relations to give the seventh condition.

The conditions yield the following relations:

1. $1+A_{2}+A_{4}=0$

2. $\left(1-\epsilon_{1} D_{1}^{2}\right)\left(A_{1} \sinh X_{1 a}+A_{2} \cosh X_{1 a}\right)$ $+\left(1-\epsilon_{1} D_{2}^{2}\right)\left(A_{3} \sinh X_{2 a}+A_{4} \cosh X_{2 a}\right)=-1$

3. $A_{L}=\left(1-\epsilon_{1} D_{1}^{2}\right) A_{2}+\left(1-\epsilon_{1} D_{2}^{2}\right) A_{4}$

4. $A_{1} \sinh X_{1 a}+A_{2} \cosh X_{1 a}+A_{3} \sinh X_{2 a}$ $+A_{4} \cosh X_{2 a}-A_{R} e^{-a / \lambda_{1}}=0$

5. $A_{L}=\left(2-\epsilon_{1} D_{1}^{2}\right) D_{1} A_{1}+\left(2-\epsilon_{1} D_{2}^{2}\right) D_{2} A_{3}$

6. $\left(2-\epsilon-\epsilon D_{1}^{2}\right) D_{1}\left(A_{1} \cosh X_{1 a}+A_{2} \sinh X_{1 a}\right)$ $+\left(2-\epsilon-\epsilon D_{2}^{2}\right) D_{2}\left(A_{3} \cosh X_{2 a}+A_{4} \sinh X_{2 a}\right)$ $+\left(\lambda_{2} / \lambda_{1}\right) A_{R} e^{-a / \lambda_{1}}=0$

7. $A_{L}+\left[\left(2-\epsilon_{1} D_{1}^{2}\right) / D_{1}\right]\left[A_{1}\left(\cosh X_{1 a}-1\right)+A_{2} \sinh X_{1 a}\right]$ $\left.+\left[2-\epsilon_{1} D_{2}^{2}\right) / D_{2}\right]\left[A_{3}\left(\cosh X_{2 a}-1\right)+A_{4} \sinh X_{2 a}\right]$ $+\left(\lambda_{1} / \lambda_{2}\right) A_{R} e^{-a / \lambda_{1}}=-a / \lambda_{2}$

where $\epsilon_{1}=\epsilon(1-\epsilon), X_{1 a}=D_{1} a / \lambda_{2}, X_{2 a}=D_{2} a / \lambda_{2}$.

Because the system is transcendental in $a$, we substitute trial values of $a$ into relations 2 to 7 and solve for the values of the $A$ 's. Then these values are substituted into 1 to test whether they satisfy that equation, too. By iterating we obtain a solution for the entire system. For $\rho_{2}=1.000, \rho_{1}=0.996$ the value of $a$ is $0.10743 \lambda_{2}$. The corresponding value of $r$ is $0.0642 \lambda_{2}$. The results for $h_{1}, h_{2}, T_{1}=v_{1} h_{1}, T_{2}=v_{2} h_{2}$ and $T_{B}=T_{1}+T_{2}$ are shown graphically in Figure 1. The results of greatest interest occur in and near the central region which has a width of the order of the internal radius of deformation, i.e., $\lambda_{i}=$ $g \epsilon \mathrm{H}_{2} / f^{2}$ which is much smaller than the barotropic radius of deformation, $\lambda_{2}$. Hence the elevations and the transports in the right and left regions will have adjustment scales considerably larger than the horizontal scale shown in Figure 1. Transports are shown relative to the maximum value of $T_{1}$.
The largest values of $T_{B}$, both positive and negative, lie within the central region and are comparable to the amplitudes of the individual transports, $T_{1}$ and $T_{2}$. The largest positive value of $T_{B}$ occurs at $x / \lambda_{2}=0.021$ and the largest negative value at $x / \lambda_{2}=0.087$ (the total width of the central region is 0.107$)$. The asymmetry of the pattern about the center is due to the influence of the barotropic surface mode which contributes a broad positive barotropic transport on an otherwise symmetric picture. The ratio of the maximum amplitudes (positive to negative) is about $4 / 3$, also due to the superimposed barotropic transport of the surface mode.

It is simpler to make a calculation of the same configuration with a rigid lid so that $H_{1}=H_{2}=H$. In this case only the baroclinic mode occurs but a barotropic transport is still generated locally. The calculation is the same as the one that Csanady [1978] carried out in a study of oceanic fronts. Only the central region has nonvanishing velocities. By symmetry the origin can be placed at the midpoint, where $h_{1}=h_{2}=H / 2$. The interface will intersect the surface at $x=-a / 2$ and the bottom at $x=a / 2$. The solution is expressed in terms of the parameters $P=\left[2 \rho_{2} /\left(\rho_{2}-\rho_{1}\right)^{1 / 2}\right]$ and $\lambda^{2}=g H / f^{2}$, which, to $0\left(\Delta \rho / \rho_{2}\right)$, are the same as $D_{1}$ and $\lambda_{2}{ }^{2}$, respectively, of the free surface case. Thus
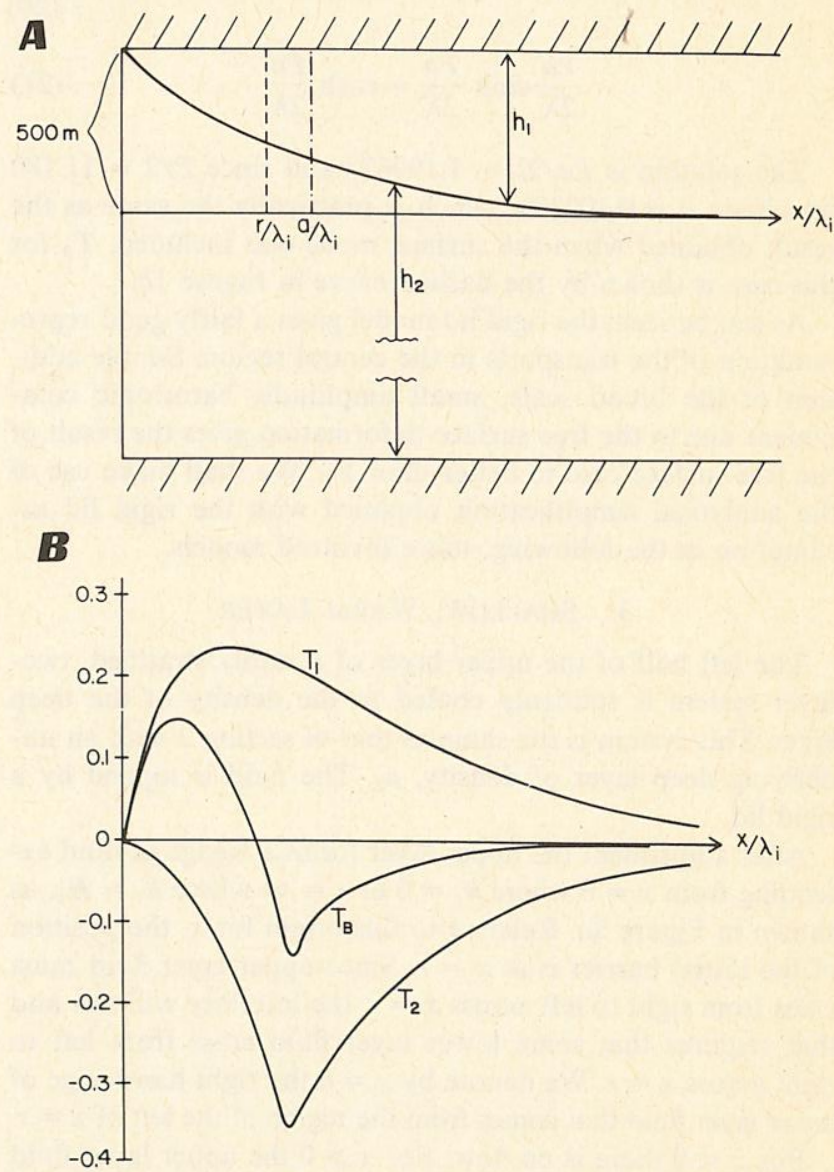

Fig. 2. (a) The shape of the interface between fluids of density $\rho_{2}$ at left and $\rho_{1}\left(=0.996 \rho_{2}\right)$ at right initially separated by a vertical barrier at $x=r$ and extending down to depth $H_{1}$. Fluid of density $\rho_{2}$ initially everywhere below $H_{1}$. Rigid lid at top. Upper layer fluid penetrates to left to point $x=0$ where $h_{1}=0$. Abscissa in units of $x / \lambda_{i}$. (b) Upper layer, lower layer, and total (barotropic) transports marked by $T_{1}, T_{2}$, and $T_{B}$ in units of $f \lambda_{i}{ }^{2} H_{1}$ for case with $H_{1}=500 \mathrm{~m}$ and $H_{2}=$ $3500 \mathrm{~m}$. 


$$
\begin{aligned}
& h_{1}=\frac{H}{2}\left(1+\frac{\sinh D x / \lambda}{\sinh D a / 2 \lambda}\right) \\
& h_{2}=\frac{H}{2}\left(1-\frac{\sinh D x / \lambda}{\sinh D a / 2 \lambda}\right)
\end{aligned}
$$

This solution brings out more clearly the role played by the internal radius of deformation, $\lambda / P=g^{\prime} H / 2 f^{2}$, where $g^{\prime}=$ $g \Delta \rho / \rho_{2}$

To find $a$ we note that there is no velocity in the lower layer at $x=-a / 2$ and no velocity in the upper layer at $x=a / 2$. Integrating the respective vorticities from these points to $x=0$ we obtain

$$
\begin{aligned}
-v_{1}(0) & =\frac{f}{H} \int_{0}^{a / 2} h_{2} d x \\
v_{2}(0) & =\frac{f}{H} \int_{-a / 2}^{0} h_{1} d x
\end{aligned}
$$

Because of symmetry at $x=0$, the sum of these must vanish so that

$$
\begin{gathered}
{\left[-x+\frac{\lambda}{P} \frac{\cosh P x / \lambda}{\sinh P a / 2 \lambda}\right]_{0}^{a / 2}-\left[x+\frac{\lambda}{P} \frac{\cosh P x / \lambda}{\sinh P x / 2 \lambda}\right]_{-a / 2}^{0}=0} \\
\frac{P a}{2 \lambda} \sinh \frac{P a}{2 \lambda}=\cosh \frac{P a}{2 \lambda}
\end{gathered}
$$

The solution is $P a / 2 \lambda=1.199678$ and since $P / 2=11.180$ we obtain $a=0.10730 \lambda$, which is practically the same as the result obtained when the surface mode was included. $T_{B}$ for this case is shown by the dashed curve in Figure $1 b$.

As can be seen, the rigid lid model gives a fairly good representation of the transports in the central region. Simple addition of the broad scale, small amplitude, barotropic component due to the free surface deformation gives the result of the free surface case to better than $1 \%$. We shall make use of the analytical simplification obtained with the rigid lid assumption in the following, more involved models.

\section{Shallow, Warm Layer}

The left half of the upper layer of a stably stratified, twolayer system is suddenly cooled to the density of the deep layer. This system is the same as that of section 2 with an underlying deep layer of density, $\rho_{2}$. The fluid is topped by a rigid lid.

After adjustment the upper layer forms a wedge of fluid extending from $x=0$ where $h_{1}=0$ to $x=\infty$ where $h_{1}=H_{1}$, as shown in Figure $2 a$. Relative to this origin for $x$, the position of the initial barrier is at $x=r$. Since upper layer fluid must cross from right to left across $x=r$, the interface will rise and that requires that some lower layer fluid cross from left to right across $x=r$. We denote by $x=a$ the right hand edge of lower layer fluid that comes from the region of the left of $x=r$.

For $x<0$ there is no flow. For $x>0$ the upper layer fluid satisfies conservation of potential vorticity given by $(1 a)$. In the region $0 \leq x \leq a$, the flow in the lower layer is governed by

$$
\frac{\zeta_{2}+f}{h_{2}}=\frac{f}{H} \quad H=H_{1}+H_{2}
$$

and for $x \geq a$ the corresponding relation is $(1 b)$.

The geostrophic equation for the upper layer is

$$
f v_{1}=\frac{1}{\rho_{1}} \frac{\partial p_{0}}{\partial x}
$$

and for the lower layer

$$
f v_{2}=\frac{1}{\rho_{2}} \frac{\partial p_{0}}{\partial x}+g^{\prime} \frac{\partial h_{2}}{\partial x}
$$

where $p_{0}$ is the pressure exerted by the lid and $g^{\prime}$ is $g \Delta \rho / \rho_{2}$.

The solution for $0 \leq x \leq a$ is

$$
\begin{gathered}
h_{1}=\frac{H_{1} H}{H_{1}+H}\left[1+b \sinh a_{1} x+c \cosh a_{1} x\right] \\
\lambda_{i} \alpha_{1}=\left(\frac{H_{1}+H}{H}\right)^{1 / 2}
\end{gathered}
$$

and for $x \geq a$

$$
h_{1}=H_{1}\left(1+d e^{-\alpha_{2} x}\right) \quad \lambda_{i} \alpha_{2}=\left(\frac{H}{H_{2}}\right)^{1 / 2}
$$

where $\lambda_{i}^{2}=g^{\prime} H_{1} / f^{2}$.

To determine the unknown amplitudes $b, c$ and $d$ and the position $a$, we use the following conditions:

1. $h_{1}(0)=0$ which yields $c=-1$.

2. The $h_{1}$ 's in (25) and (26) are equal at $x=a$.

3. The Margules relations across $x=a$ are matched, i.e., $h_{1 x}$ must be continuous at $x=a$. This is equivalent to requiring continuity of the $v$ 's at $x=a$.

4. The mass of upper layer fluid in $x \leq r$ must equal the mass of lower layer fluid above $\mathrm{H}_{2}$ and to the right of $r$. Although it is not immediately obvious, condition 4 is equivalent to the statement $v_{2}(0)=0$, which gives continuity of $v_{2}$ across $x=0$. Integration of the potential vorticity equation (22) from 0 to $x$ and of $(1 b)$ from $x$ to $\infty$ yield

$$
\begin{gathered}
v_{2}=v_{2}(0)+f \int_{0}^{x} \frac{h_{2}-H}{H} d x \\
v_{2}=\int_{x}^{\infty} \frac{h_{2}-H_{2}}{H_{2}} d x
\end{gathered}
$$

and when the $v_{2}$ 's are matched at $x=a$, it can be seen that matching of the integrals (required by 4 ) leads to $v_{2}(0)=0$ and vice versa.

The results for this case with $\rho_{1}=0.996, \rho_{2}=1.0$ and $H_{2}=$ $7 H_{1}$ (corresponding to $H_{1}=500 \mathrm{~m}$ and $H_{2}=3500 \mathrm{~m}$ ) are shown in Figure 2. The interface is at depth $H_{1}$ at large $x$ and rises to the surface at $x=0$. The positions $r$ and $a$ are given by $a \alpha_{1}=1.09$ and $r \alpha_{1}=0.901$. A barotropic transport of about 6 Sverdrups flows in the positive direction (dense water to the left) in the interval $0 \leq x \leq 0.8 a \alpha_{1} \lambda_{i}$ and an equal but opposite transport with peak negative value at $x=1.05 a \alpha_{1} \lambda_{i}$ occupies the region to the right. The upper (lower) layer transport is everywhere positive (negative) and amounts to about 35 Sverdrups.

The total barotropic transport from $x=0$ to $\infty$ vanishes. This result can be obtained by integrating the solutions given above from 0 to $\infty$ but a simple expression for it is easier to obtain from the original equations. Thus substituting $h_{1}=$ $H_{1} v_{1 x} / f+H_{1}$ in $h_{1} v_{1}$ to obtain

$$
\int_{0}^{a} h_{1} v_{1} d x=\frac{H_{1}}{2 f}\left[v_{1}^{2}(a)-v_{1}^{2}(0)\right]+\int_{0}^{a} H_{1} v_{1} d x
$$

and $h_{2}=H_{2} v_{2 x} / f+H$ in $h_{2} v_{2}$ to obtain 
A

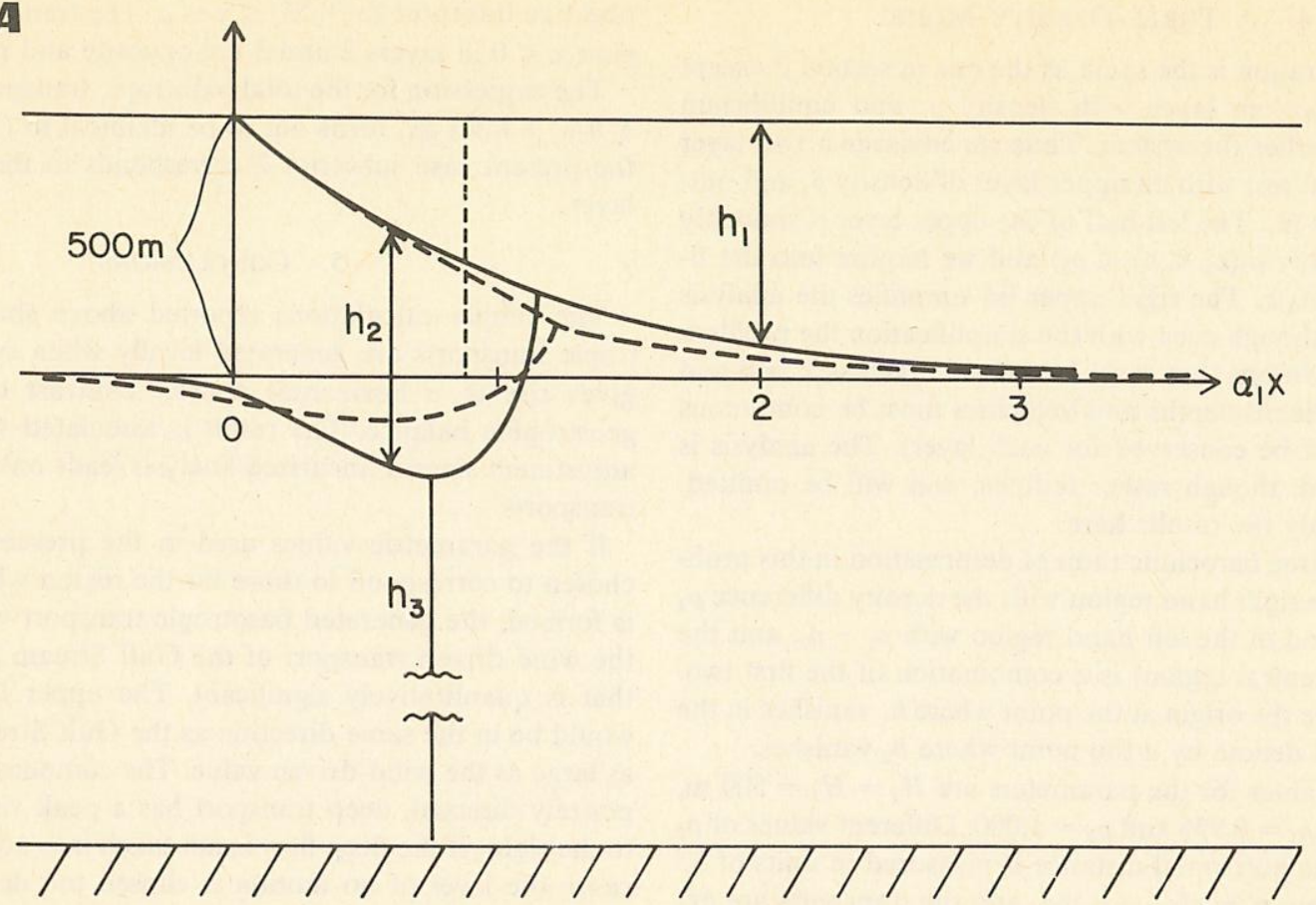

B

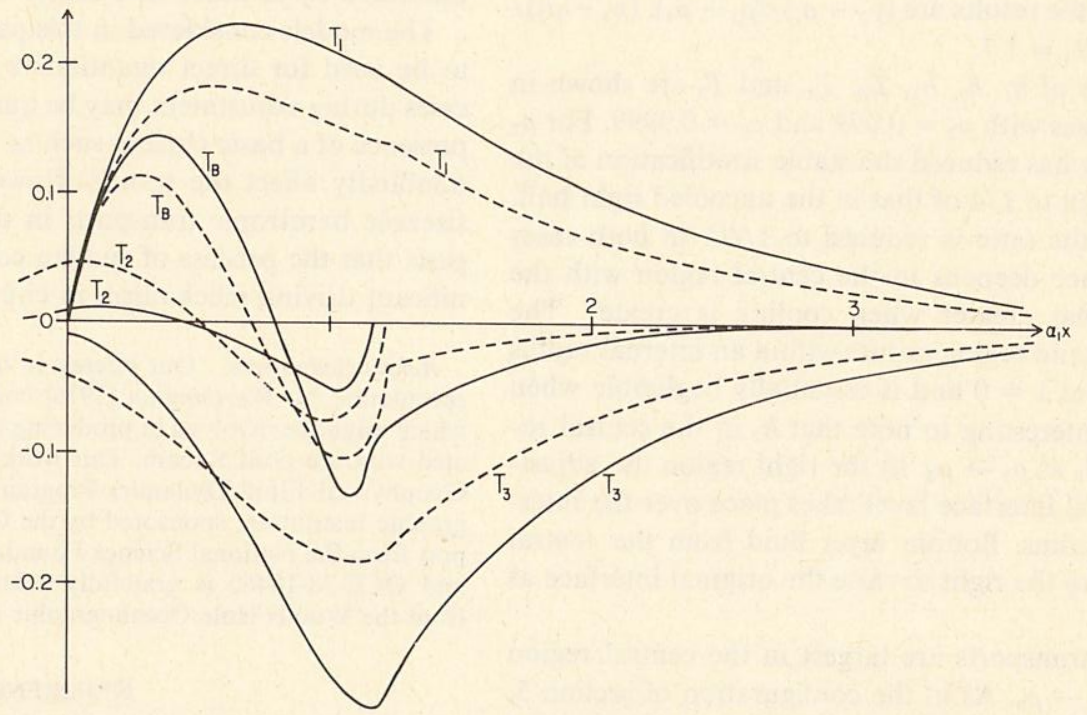

Fig. 3. (a) The interfaces between fluids of densities $\rho_{1}\left(=0.996 \rho_{3}\right), \rho_{2}$, and $\rho_{3}$ for the three density cases with a rigid lid. Abscissa measured in units of $\alpha_{1} x$ from point where $h_{1}$ vanishes. Solid curves for $\rho_{2}=0.999 \rho_{3}$ and dashed curves for $\rho_{2}$ $=0.9999 \rho_{3}$. In latter case thickness $h_{2}$ is nearly constant $($ at $500 \mathrm{~m})$ to point where it vanishes. $(b)$ Transports for the three layers for the two values of $\rho_{2}$ of Figure $3 a$. Also shown is $T_{B}$ for each case. For $\rho_{2}=0.9999 \rho_{3}$ results are nearly identical to those of Figure 2.

$$
\int_{0}^{a} h_{2} v_{2} d x=\frac{H}{2 f}\left[v_{2}^{2}(a)-v_{2}^{2}(0)\right]+\int_{0}^{a} H_{2} v_{2} d x
$$

and making use of

$$
\int_{0}^{a}\left(H_{1} v_{1}+H_{2} v_{2}\right) d x=a H_{1} v_{1}(0)+a H_{2} v_{2}(0)-\frac{f H_{1} a^{2}}{2}
$$

yield, with $v_{2}(0)=0$,

$$
\begin{aligned}
\int_{0}^{a}\left(h_{1} v_{1}+h_{1} v_{2}\right) d x= & \frac{H_{1}}{2 f}\left[v_{1}^{2}(a)-v_{1}^{2}(0)\right] \\
& +\frac{H}{2 f} v_{2}^{2}(a)+a H_{1} v_{1}(0)-\frac{f H_{1} a^{2}}{2}
\end{aligned}
$$

In a similar fashion

$$
\int_{a}^{\infty}\left(h_{1} v_{1}+h_{2} v_{2}\right) d x=-\frac{H_{1}}{2 f} v_{1}^{2}(a)-\frac{H_{2}}{2 f} v_{2}^{2}(a)
$$

The sum of (28) and (29) yields

$$
\int_{0}^{\infty}\left(h_{1} v_{1}+h_{2} v_{2}\right) d x=H_{1}\left\{a v_{1}(0)+\frac{v_{2}^{2}(0)-v_{1}^{2}(0)}{2 f}-\frac{f a^{2}}{2}\right\}
$$

which is easy to evaluate and turns out to vanish for any particular case. 


\section{A ThreE-DENSITY MODEL}

The configuration is the same as the one in section 2 except that a deep bottom layer with density $\rho_{3}$ and equilibrium depth $H_{3}$ underlies the system. Thus we envisage a two-layer fluid initially at rest with an upper layer of density $\rho_{1}$ and uniform thickness $H_{1}$. The left half of the upper layer is suddenly cooled to density $\rho_{2}\left(\rho_{1}<\rho_{2}<\rho_{3}\right)$ and we inquire into the final, adjusted state. The rigid upper lid simplifies the analysis considerably, though even with the simplification the problem has seven conditions that must be satisfied (across $x=0$ and $x=a$ the pertinent depths and velocities must be continuous and mass must be conserved for each layer). The analysis is straightforward, though rather tedious, and will be omitted. We present only the results here.

There are three baroclinic radii of deformation in this problem, one in the right hand region with the density difference $\rho_{3}$ $-\rho_{1}$, the second in the left hand region with $\rho_{3}-\rho_{2}$, and the third (in the central region) is a combination of the first two. Again we place the origin at the point where $h_{1}$ vanishes in the final state and denote by $a$ the point where $h_{2}$ vanishes.

As before values for the parameters are $H_{1}=H_{2}=500 \mathrm{~m}$, $H_{3}=3500 \mathrm{~m}, \rho_{1}=0.996$ and $\rho_{3}=1.000$. Different values of $\rho_{2}$ were used. The horizontal distance is measured in units of $\lambda_{i}{ }^{2}$ $=g^{\prime} H_{1} / f^{2}$ where $g^{\prime}=g\left(\rho_{3}-\rho_{1}\right) / \rho_{3}$ and the transports are expressed in units of $H_{1} \lambda_{i} f$. As a consequence, the only parameters that enter into the results are $\left(\rho_{2}-\rho_{1}\right) /\left(\rho_{3}-\rho_{1}\right),\left(\rho_{3}-\rho_{2}\right) /$ $\left(\rho_{3}-\rho_{1}\right)$ and $H_{1} / H_{3}=1.7$.

The distributions of $h_{1}, h_{2}, h_{3}, T_{B}, T_{2}$, and $T_{3}$ are shown in Figure 3 for the cases with $\rho_{2}=0.999$ and $\rho_{2}=0.9999$. For $\rho_{2}$ $=0.999$ the cooling has reduced the stable stratification of the left half of the ocean to $1 / 4$ of that in the uncooled right half. With $\rho_{2}=0.9999$ the ratio is reduced to $1 / 40$. In both cases the original interface deepens in the central region with the deepening somewhat greater when cooling is greater. The change in the left hand region occurs within an internal radius of deformation from $x=0$ and is essentially negligible when $\rho_{2}=0.9999$. It is interesting to note that $h_{2}$ in the central region approaches $H_{2}$ as $\rho_{2} \rightarrow \rho_{3}$. In the right region the adjustment to this original interface level takes place over the internal deformation radius. Bottom layer fluid from the central region has moved to the right to raise the original interface as shown.

The barotropic transports are largest in the central region and increase as $\rho_{2} \rightarrow \rho_{3}$. As in the configuration of section 3 , the maximum positive $T_{B}$ occurs in the left part of the central region and $T_{B}>0$ in nearly the entire central part. The negative barotropic transport is of smaller amplitude, reaches its maximum near $x_{1}=1$ and extends farther into the right hand region.

The results for the case with $\rho_{2}=0.9999$ are practically the same as for the two-density case considered in section 3 . With $\rho_{2}=0.999$ the positive (negative) barotropic transport in the central (right) region is about 4 Sverdrups. The upper (lower) layer transport is again positive (negative) everywhere. The intermediate layer (2) has positive transport for $x \leqq 0.5 \lambda_{i}$ and negative transport for $0.5 \lambda_{i} \leq x \leq a$. The transports in the region $x<0$ in layers 2 and 3 are opposite and nearly equal.

The expression for the total barotropic transport, $\int_{-\infty}^{\infty}\left(h_{1} v_{l}\right.$ $\left.+h_{2} v_{2}+h_{3} v_{3}\right) d x$, turns out to be identical to (30), though in the present case subscript 2 corresponds to the intermediate layer.

\section{CONCLUSIONS}

The simple calculations reported above show that barotropic transports are generated locally when sudden cooling gives rise to a horizontal density contrast that is not in geostrophic balance. The result is associated with nonlinear adjustment since a linearized analysis leads only to baroclinic transports.

If the parametric values used in the present analysis are chosen to correspond to those for the region where $18^{\circ}$ water is formed, the generated barotropic transport would enhance the wind-driven transport of the Gulf Stream by an amount that is quantitatively significant. The upper layer transport would be in the same direction as the Gulf Stream and about as large as the wind-driven value. The compensating, and oppositely directed, deep transport has a peak value somewhat to the right. If the deep flow is not taken into account, e.g., because the level of no motion is chosen too deep, the downstream barotropic transport of the Gulf Stream could be overestimated by as much as a factor of 3 .

The models considered in this paper are much too idealized to be used for direct quantitative estimates. Transient processes during adjustment may be quantitatively significant. The presence of a basic current such as the Gulf Stream would undoubtedly affect the results. However, the demonstration of sizeable barotropic transports in these idealized models suggests that the process of sudden cooling be included as a significant driving mechanism in circulation models.

Acknowledgments. Our interest in this problem was stimulated by speculation by Worthington [1976] concerning possible mechanisms which might be involved in producing the recirculation regime associated with the Gulf Stream. This work was initiated during the 1979 Geophysical Fluid Dynamics Program at the Woods Hole Oceanographic Institution, sponsored by the Office of Naval Research. Support from the National Science Foundation via grants OCE-77-19451 and OCE-78-18460 is gratefully acknowledged. Contribution 4665 from the Woods Hole Oceanographic Institution.

\section{REFERENCES}

Csanady, G. T., Wind effects on surface to bottom fronts, J. Geophys. Res., 83, 4633-4640, 1978.

Rossby, C.-G., In the mutual adjustment of pressure and velocity distribution in certain simple current systems, II, J. Mar. Res., 1, 239$263,1938$.

Worthington, L. V., On the North Atlantic circulation, Oceanogr. Stud. 6, 110 pp., The Johns Hopkins Univ., Baltimore, Md., 1976.

(Received February 19, 1980; revised May 2, 1980; accepted May 2, 1980.) 


\section{MANDATORY DISTRIBUTION LIST}

FOR UNCLASSIFIED TECHNICAL REPORTS, REPRINTS, AND FINAL REPORTS PUBLISHED BY OCEANOGRAPHIC CONTRACTORS

OF THE OCEAN SCIENCE AND TECHNOLOGY DIVISION

OF THE OFFICE OF NAVAL RESEARCH

(REVISED NOVEMBER 1978)

1 Deputy Under Secretary of Defense (Research and Advanced Technology) Military Assistant for Environmental Science Room 30129

Washington, D.C. 20301

Office of Naval Research 800 North Quincy Street

Arlington, VA 22217

3 ATTN: Code 483

1 ATTN: Code 460

2 ATTN: 102B

1 CDR J. C. Harlett, (USN) ONR Representative Woods Hole Oceanographic Inst. Woods Hole, MA 02543

Commanding Officer Naval Research Laboratory Washington, D.C. 20375

6 ATTN: Library, Code 2627
12 Defense Documentation Center Cameron Station Alexandria, VA 22314 ATTN: DCA

Commander Naval Oceanographic Office NSTL Station

Bay St. Louis, MS 39522

ATTN: Code 8100

ATTN: Code 6000

ATTN: Code 3300

1 NODC/NOAA

Code D781

Wisconsin Avenue, N.W. Washington, D.C. 20235 


\begin{tabular}{|c|c|}
\hline REPORT DOCUMENTATION PAGE & $\begin{array}{l}\text { READ INSTRUCTIONS } \\
\text { BEFORE COMPLETING FORM }\end{array}$ \\
\hline $\begin{array}{l}\text { 1. REPORT NUMBER } \\
\text { WHOI }-81-22\end{array}$ & 3. RECIPIENT'S CATALOG NUMBER \\
\hline $\begin{array}{l}\text { 4. TITLE (and Subtitie) } \\
\text { BAROTROPIC RESPONSE TO COOLING }\end{array}$ & $\begin{array}{l}\text { 5. TYPE OF REPORT \& PERIOD COVERED } \\
\text { TECHNICAL }\end{array}$ \\
\hline BAROTROPIC RESPONSE TO COOLING & $\begin{array}{l}\text { 6. PERFORMING ORG. REPORT NUMBER } \\
\text { WHOI Cont. } 4665\end{array}$ \\
\hline $\begin{array}{l}\text { 7. AUTHOR(o) } \\
\text { Henry Stommel and George Veronis }\end{array}$ & $\begin{array}{l}\text { 8. CONTRACT OR GRANT NUMBER(ब) } \\
\text { NO0014-79-C-0671 } \\
\text { OCE 77-19451 } \\
\text { OCE 78-18460 }\end{array}$ \\
\hline $\begin{array}{l}\text { 9. PERFORMING ORGANIZATION NAME AND ADDRESS } \\
\text { Woods Hole Oceanographic Institution } \\
\text { Woods Hole, Massachusetts } 02543\end{array}$ & $\begin{array}{l}\text { 10. PROGRAM ELEMENT, PROJECT, TASK } \\
\text { AREA \& WORK UNIT NUMBERS }\end{array}$ \\
\hline \multirow{2}{*}{$\begin{array}{l}\text { 11. CONTROLLING OFFICE NAME AND ADDRESS } \\
\text { NORDA/National Space Technology Laboratory } \\
\text { Bay St. Louis, MS } 39529\end{array}$} & $\begin{array}{l}\text { 12. REPORT DATE } \\
\text { April } 1981\end{array}$ \\
\hline & 13. NUMBER OF PAGES \\
\hline \multirow[t]{2}{*}{ 14. MONITORING AGENCY NAME \& ADDRESS(If different from Controlling Office) } & $\begin{array}{l}\text { 15. SECURITY CLASS. (of thie roport) } \\
\text { UNCLASSIFIED }\end{array}$ \\
\hline & $\begin{array}{l}\text { 15a. DECLASSIFICATION/DOWNGRADING } \\
\text { SCHEDULE }\end{array}$ \\
\hline
\end{tabular}

16. DISTRIBUTION STATEMENT (of this Report)

Approved for public release; distribution unlimited.

17. DISTRIBUTION STATEMENT (of the abotract ontored in Block 20, if different from Roport)

18. SUPPLEMENTARY NOTES

Reprinted from: Journal of Geophysical Research 85(C11): 6661-6666 (November 20, 1980).

19. KEY WOROS (Continue on reverae olde if neceesary and identify by block number)

1. Barotropic response

2. Cooling

3. Density forcing

20. ABSTRACT (Continue on reverae aldo if necoesery and identify by block number)

See reverse side. 
20.

Imposed horizontal density differences in a nonrotating fluid generate vertical circulation which has vanishing vertically integrated transports. When the system is rotating, geostrophic velocities can balance the density differences and the vertically integrated transports need not vanish locally. In a two-layer fluid, finite amplitude disturbances lead to barotropic flows that have the same direction as the velocity in the layer that thickens as a result of the disturbance. Specific calculations are carried out for the geostrophic adjustment model in situations that approximate those in which $18^{\circ}$ water is formed south of the Gulf Stream. The upper layer transport that results from sudden cooling (as simulated by density differences that are initially unbalanced geostrophically) is in the same direction as the Gulf Stream transport and comparable to it in magnitude. A lower level transport of the same magnitude flows in the opposite direction with a maximum value about an internal radius of deformation to the right of that of the upper layer. The barotropic transport is about $1 / 5$ as large and flows downstream in the Gulf Stream and upstream to the right of the Gulf Stream. 

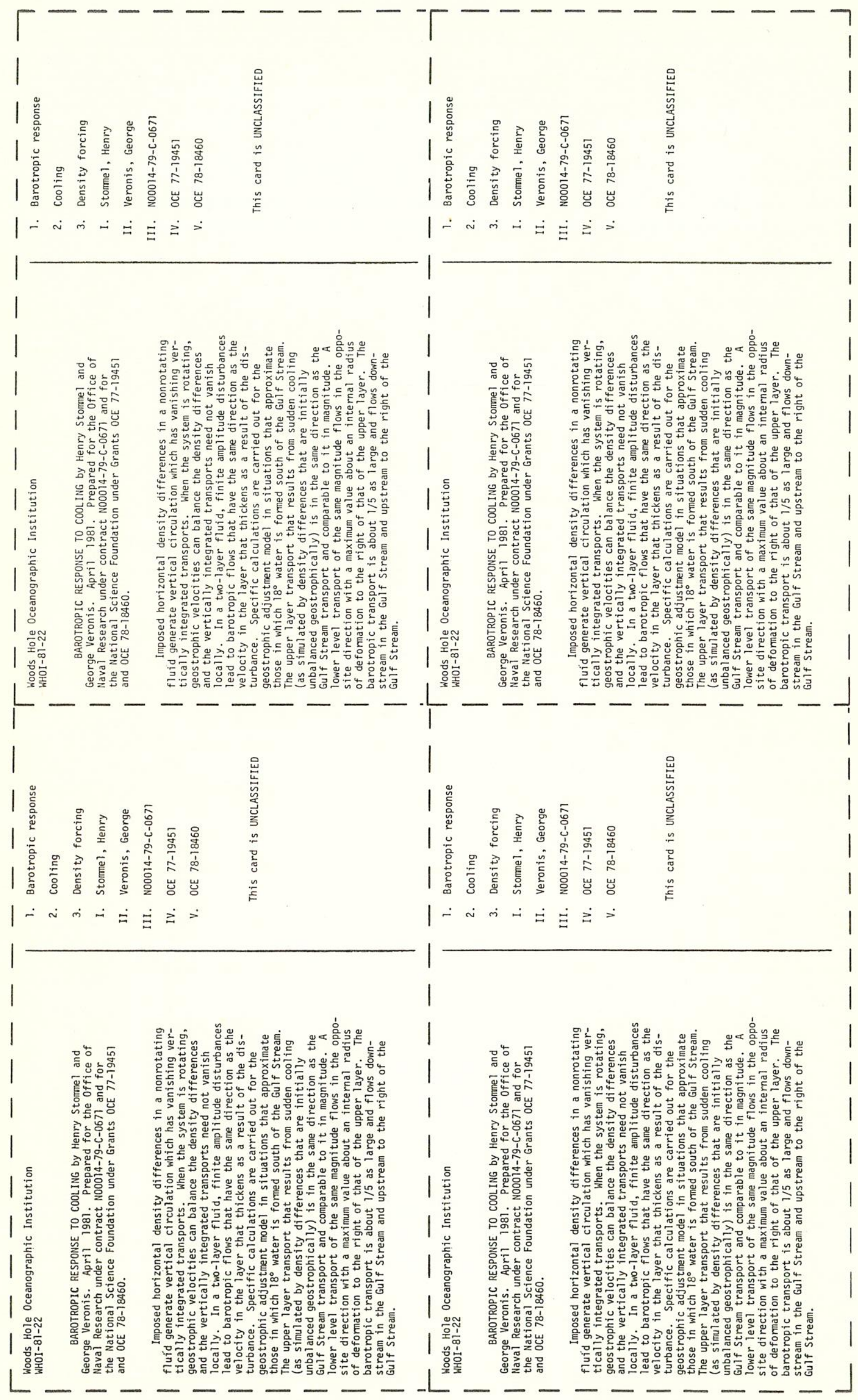